\title{
ECONOMII
}

Revista Economía y Política

ISSN: 1390-7921

revista.economiaypolitica@ucuenca.edu.ec

Universidad de Cuenca

Ecuador

\section{Factores explicativos del crecimiento del sector público. El caso de Ecuador 1983-2016}

Matute Pacheco, Juan B.

Factores explicativos del crecimiento del sector público. El caso de Ecuador 1983-2016

Revista Economía y Política, vol. XV, núm. 30, 2019

Universidad de Cuenca, Ecuador

DOI: https://doi.org/10.25097/rep.n30.2019.08

(C) Universidad de Cuenca 2019

Esta obra está bajo una Licencia Creative Commons Atribución-NoComercial-Compartirlgual 4.0 Internacional. 


\section{Factores explicativos del crecimiento del sector público. El caso de Ecuador}

\section{$1983-2016$}

\section{Explanatory factors of public sector growth. The case of Ecuador 1983-2016}

Juan B. Matute Pacheco

DOI: https://doi.org/10.25097/rep.n30.2019.08

\section{ETAPA EP, Ecuador}

juanbernardo.matute@gmail.com

\section{Resumen:}

El presente trabajo examina los determinantes del crecimiento del sector público en Ecuador en el período 1983-2016 bajo cinco teorías explicativas: Ley de Wagner, internacionalización económica, burocracia, ilusión fiscal e ideología del partido político. Los resultados revelan evidencia fuerte de que el crecimiento del sector público en Ecuador está asociado a factores de orden político e institucional, como la ideología del partido de gobierno y los efectos de la burocracia sobre el sistema democrático. Por el contrario, se encontró apoyo parcial a favor de la Ley de Wagner y la internacionalización de la economía y ningún soporte a la tesis de que el crecimiento del sector público se debe a la ilusión fiscal creada por el sistema impositivo. El análisis comparativo entre países muestra evidencia de que el tamaño del sector público en Ecuador está sobredimensionado en relación a otros países con similares niveles de desarrollo.

Palabras clave: Crecimiento económico, Ilusión Fiscal, instituciones, Ley de Wagner, sector público.

\section{Abstract:}

The following research examines the determining factors of the growth of the public sector in Ecuador in the period between 1983-2016, using five explanatory theories: Wagner's Law, economic internationalization, bureaucracy, fiscal illusion and political party ideology. The results reveal strong evidence that the growth of the public sector in Ecuador is linked political and institutional factors such as the the governing party's ideology and the effects of bureaucracy on the democratic system. On the opposite side of the spectrum, partial support for Wagner's Law and economic internationalization was found and no support for the theory that the growth of the public sector is due to fiscal illusion created by the tax system. The comparative analysis between countries presents evidence that the size of the public sector in Ecuador is oversized in relation to countries with similar levels of development.

KEYWORDS: Economic growth, Fiscal illusion, institutions, public sector, Wagner's law.

\section{INTRODUCCIÓN}

Una de las características que ha marcado la senda de las economías desarrolladas durante el último siglo ha sido el constante crecimiento y expansión del sector público, y la consolidación del Estado del Bienestar. En países como Suecia, paradigma del Estado de Bienestar europeo, o Alemania, el sector público pasó del 8,72\% y $16,34 \%$ del PIB en el año 1930 a $47,87 \%$ y $43,42 \%$ en el 2015 , respectivamente ${ }^{[1]}$. El mundo en desarrollo parece no haber sido ajeno a esta realidad y algunos países de Latinoamérica han visto al estado de bienestar de los países europeos como un faro político a emular como medio para alcanzar mayores niveles de desarrollo, bienestar y cohesión social. Particularmente en Ecuador, el sector público ha crecido de forma acelerada del 11,57\% del PIB en el año 1983 hasta llegar a sobrepasar el 40\% en el 2014 y el 36,8\% en el 2016, muy por encima del crecimiento del PIB per cápita ${ }^{[2]}$ que para el mismo período fue del 1,13\%, situación que lo acerca 
a países desarrollados respecto al tamaño del sector público pero muy por debajo en cuanto a resultados y estándares de desarrollo económico y social.

Ante este escenario de rápida expansión de la participación del sector público en relación al total de recursos de la economía, sin el correspondiente retorno en cuanto a crecimiento económico, el presente trabajo busca dar respuesta a la pregunta de investigación ¿Cuáles son los factores o determinantes que han incidido en el crecimiento del sector público en la economía de Ecuador, en el período 1983-2016? Adicionalmente, entorno a la pregunta principal, se plantean las siguientes preguntas particulares: 1) ¿Está sobredimensionado el tamaño del sector público de Ecuador en comparación a otros países con similares niveles de desarrollo medido por su PIB per cápita? y 2) ¿Existe relación de causalidad o de equilibrio a largo plazo entre crecimiento del sector público y crecimiento económico en la economía de Ecuador?

El determinar las causas asociadas a la expansión del sector público es prioritario ya que, dependiendo de la génesis y factores asociados a este crecimiento, se originan efectos en el crecimiento económico, en la redistribución del ingreso, en la carga fiscal que ejerce el financiamiento de las actividades estatales sobre los contribuyentes y consecuentemente, en la capacidad de generación de renta de las economías en desarrollo para sostener un elevado nivel de gasto público en un entorno de baja calidad institucional que limita su beneficio potencial e incrementa los riesgos asociados a su gestión (corrupción, ineficiencia, etc.).

\section{Revisión de la Literatura: Teorías explicativas Sobre el CRECimiento del Sector PÚBLICO}

\subsection{El crecimiento del sector público en la literatura teórica}

De manera general, siguiendo a Boix (2011), las teorías explicativas del crecimiento del sector público pueden agruparse en tres grandes grupos: 1) Teorías por el lado de la demanda, 2) Teorías institucionalistas o de oferta, 3) Teorías políticas o de redistribución.

\subsubsection{Teorías explicativas por el lado de la demanda}

Las teorías por el lado de la demanda enfatizan que el crecimiento del sector público es consecuencia natural del propio crecimiento económico o de variables económicas, por lo que conciben al sector público como un proveedor de servicios públicos. Dentro de este enfoque están: a) Teoría del Industrialismo o Ley de Wagner; b) Internacionalización de la Economía.

Teoria del Industrialismo o Ley de Wagner

Uno de los pioneros en el estudio del crecimiento del sector público fue el filósofo alemán Adolph Wagner quien en el año 1877 postuló lo que el mismo denominó "ley de expansión creciente de las actividades públicas y en particular de las actividades estatales".

Postulada en medio de un período de rápida urbanización e industrialización, y justo en la época en que Bismark estaba desarrollando su primer programa del Estado de Bienestar, Wagner reconoció el rol del estado como proveedor de gasto en inversión social, educación y transporte, etc., incluso en democracias autoritarias, para de esta forma mantener la legitimidad social del estado por medio de fondos públicos destinados a compensar los costos humanos del desarrollo económico. (Cameron, 1978, pág. 1245).

Específicamente, la ley de Wagner afirma que, a medida que el ingreso agregado y el ingreso per cápita de una nación se incrementan y el país se vuelve más rico, el tamaño relativo del sector público respecto al ingreso total de la economía también se incrementa inevitablemente, de forma que existiría una relación de causalidad, en donde los incrementos del ingreso (total o per cápita) generan el aumento del gasto público y no viceversa.

Este hecho tiene importantes consecuencias en materia de política económica; según Magazzimo, (2012), la Ley de Wagner contradice la hipótesis Keynesiana, ya que, si la causalidad va del ingreso al gasto público, 
este último no puede ser usado como un instrumento exógeno para incidir en el crecimiento económico y en el ingreso agregado, tal como lo establece la teoría keynesiana, puesto que según Wagner, el gasto público es una variable endógena determinada por factores puramente económicos y consecuentemente, la política económica de manejo del gasto público tendría una débil capacidad para incidir en el crecimiento.

Una de las críticas que recibe el enfoque del industrialismo es el de determinismo económico; según Ochando (1999), la industrialización sería una condición necesaria pero no suficiente para la expansión del sector público y particularmente del estado de bienestar, por lo que, a más de los factores expuestos por Wagner, tendrían que considerarse otros factores políticos, institucionales y de incentivos que explican y matizan la forma como han llegado a constituirse los estados modernos.

\section{Internacionalización de la Economía}

La internacionalización económica también es considerada como la causa principal del crecimiento del sector público en las economías occidentales. La internacionalización, la apertura económica y especialmente el libre comercio puede aumentar los riesgos a los que se exponen ciertos sectores de la economía nacional, y con ello, aumentar las presiones políticas hacia el aumento del gasto público orientado a compensar a sectores afectados que no son capaces de competir con las mercancías producidas en el exterior sin ayudas arancelarias. Bajo este enfoque, a nivel macroeconómico, un alto grado de dependencia del comercio internacional aumentaría la inestabilidad de la economía ya que debilitaría el grado de control en la política económica, situación que conduce a los gobiernos a expandir su nivel de actividad para reducir el efecto de la internacionalización en la producción agregada, el empleo y el consumo.

\subsubsection{Teorías Explicativas según el Enfoque de Oferta o Institucionalista}

Las teorías por el lado de la oferta enfatizan que la expansión del gasto público, no necesariamente responde a satisfacer demandas sociales, sino más bien, obedece a la propia estructura institucional del estado donde coexisten grupos de poder con capacidad institucional para imponer sus propios intereses (burocracia). Las teorías institucionalistas de crecimiento del gasto púbico son: a) La influencia de la burocracia; b) Ilusión Fiscal.

\section{La influencia de la Burocracia}

Desde esta perspectiva, la expansión del sector público es vista como el resultado del poder de voto de los empleados públicos y su capacidad para influir en el resto de los votantes. A medida que el empleo público llega a ser una parte cada vez más importante de la fuerza de trabajo y de la población votante, la burocracia aumenta su poder de influencia en las elecciones democráticas y se crean incentivos para que los políticos desarrollen agendas que favorezcan a este grupo a fin de captar sus votos. A su vez, resultaría más probable que los burócratas tengan en promedio mayores incentivos para favorecer con su voto a aquellos candidatos con programas de expansión del gasto público en comparación con el resto de los votantes. El crecimiento del empleo generado por el sector público crea incentivos para la existencia de grupos de interés (burocracia) que presionan el gasto público al alza, motivo por el cual, el estado de bienestar es muy difícil de desmantelar una vez se ha producido su expansión.

\section{Ilusión Fiscal}

Otra hipótesis alternativa que podría explicar la expansión del sector público es la estructura específica del sistema impositivo. Se argumenta que mientras mayor es la complejidad del sistema y la participación de los impuestos indirectos en la financiación del gasto público, los contribuyentes son menos conscientes de la verdadera carga impositiva del sistema; ante la presencia de esta ilusión fiscal, los contribuyentes tienden a subestimar los costos y a sobrestimar los beneficios de los servicios públicos, demandando así, un mayor gasto público de lo socialmente necesario. Buchanan (1967), argumentó que la ilusión fiscal hace referencia al "engaño" que los poderes del Estado pueden realizar para disfrazar el verdadero tamaño estatal y sus capacidades de organizar y regular. Un sistema fiscal basado en impuestos directos es más transparente en cuanto a transmitir al contribuyente el costo de la carga fiscal y de financiación del gasto público, por lo que se espera que tienda a contener su expansión. 


\subsubsection{Teoría Política o de Redistribución}

Según Meltzer y Richards citados en (Boix, 2001), bajo este enfoque, el crecimiento del sector público responde a conflictos sociales y redistributivos entre los actores económicos. Específicamente, son dos los aspectos identificados del sistema político con influencia en la magnitud de la expansión de la economía pública y que son analizados en dos estudios realizados por Cameron (1978) y Garand (1988): La competencia electoral de los partidos políticos por captar votos y la ideología del partido de gobierno.

\section{Ideología del Partido Político en el poder y competencia electoral}

En referencia a la competencia electoral, esta incide en la expansión de la economía pública debido a que los partidos y las élites políticas se comportan de un modo racional manipulando el gasto público como estrategia para captar los votos del electorado.

La competencia electoral tiene importantes efectos y consecuencias redistributivas debido a que los políticos buscarán ganarse al electorado ofreciendo y ejecutando transferencias desde los votantes que se encuentran por encima de la renta media de la economía a favor de los que se encuentran por debajo, si consideran a estos últimos, como un nicho importante de votos. No obstante, también podría resultar regresivo, ya que una cosa son los ofrecimientos de campaña y otra el accionar de los políticos una vez que conquistan el poder, el cual podría favorecer a grupos con mayor poder político y económico, en perjuicio de los individuos de la parte baja de la distribución de renta.

Otro factor político y uno de los más controvertidos para explicar el crecimiento del sector público es la ideología del partido de gobierno en el poder dentro del sistema democrático. Bajo esta hipótesis, la expansión del estado sería el resultado de la ascensión al poder de la clase trabajadora organizada en partidos de izquierda y por el contrario, la baja participación del sector público estaría asociada a sistemas políticos donde predominan en el poder partidos de derecha, que tienden a reducir la provisión de gasto público.

Según Cameron (1978), los críticos de la tesis de la ideología sostienen que los partidos especialmente de izquierdas, han abandonado sus posiciones ideológicas para abarcar electorados más amplios y aunque mantuvieran su posición ideológica, pueden ser incapaces de implementar sus preferencias cuando llegan al poder debido a la movilización social y a la negociación política con otros partidos con los cuales comparten poder.

Según Ochando (1999), "la ideología del partido político va perdiendo fuerza explicativa de la expansión del sector público debido al impacto del estado de bienestar sobre la propia cultura. El estado de bienestar se ha convertido en un símbolo de identidad de muchos de los países más desarrollados y ha sido incorporado en la cultura democrática de las sociedades con una relativa independencia del partido de gobierno. Por tanto, el estado de bienestar al estar enraizado en la cultura democrática resulta difícil de desmontar, sea cual fuere la ideología del partido, que ostenta el poder político". (pp. 123)

\subsection{Evidencia empírica en la literatura relativa al crecimiento del sector público}

Los estudios realizados en las décadas de los 70 y 80 entre los que resaltan los de Cameron (1978), Berry \& David (1984) y Garand (1988), buscan explicar el crecimiento del sector público no solo como un fenómeno ligado al desarrollo económico, sino vinculado a factores políticos e institucionales tales como la influencia de la burocracia, la competencia electoral, ilusión fiscal, grado de descentralización gubernamental, etc.

Para el caso de Estados Unidos los trabajos de Berry \& David (1984) y Garand (1988) tienen en común en sus modelos a la ley de Wagner, la ilusión fiscal y la burocracia. Ninguno encuentra evidencia suficiente para apoyar a la Ley de Wagner como factor de crecimiento del sector público en EE.UU, en tanto que, respecto a las variables ilusión fiscal y burocracia ambos estudios llegan a resultados contradictorios.

El estudio de Cameron (1978) con datos de panel para 18 países en el período 1960 - 1975 tampoco provee evidencia suficiente que valide la ley de Wagner y la ilusión fiscal, pero si encuentra relaciones que apoyan la hipótesis de que los partidos de izquierda, el grado de centralización gubernamental y fiscal, y la internacionalización de la economía conducen a una mayor expansión del sector público. 
Como se mencionó anteriormente, si la ideología del partido político en el poder tiene o no un efecto significativo para explicar la expansión de la economía pública y de ser así, cómo lo hace, ha sido materia de intenso debate. A nivel empírico, Cameron (1978) en su estudio comparativo de las razones de la expansión del sector público, sostiene que, contrario al punto de vista de los escépticos, la política y especificamente la ideología del partido de gobierno está relacionada con la tasa de expansión del sector público, siendo esta mayor cuando el gobierno está controlado por partidos socialdemócratas y sus aliados de izquierda en comparación con partidos de derecha. Según este autor, países como Suecia, Noruega y Dinamarca, en donde los partidos de izquierda tienden en promedio a conformar la mayoria del gobierno, experimentaron incrementos mayores del tamaño del sector público, en comparación a naciones como Japón, Italia o Francia, donde la izquierda únicamente participó como aliado minoritario, o quedó excluida del gobierno. Hibbs (1976), tambien encuentra evidencia a favor de la tesis de la ideología del partido político y afirma que las huelgas disminuyeron en las naciones Europeas entre 1930 y 1940, cuando los partidos laboristas y socialdemócratas incrementaron su participación en el gobierno, debido a la mayor tendencia de estos partidos a expandir los estados de bienestar. Esta tendencia conllevó a incrementar la participación del sector público en relación al total de la economía, pero aún más importante, como señala Cameron (1978), trasladó las fricciones redistributivas del sector privado, en donde compiten capital y trabajo, al sector público en donde el conflicto se resuelve mediante la competencia de los actores económicos entre sí mismos y con el gobierno, a través de la movilizacion electoral y la negociación política. Hibbs (1977), en su análisis de la relación entre la ideológia de los partidos políticos y la política macroeconomica para 12 países de Europa Occidental y Norteamérica durante el período de postguerra, afirma que los países con predominancia de partidos de izquierda tienden a prevalecer los objetivos de empleo y la redistribución de ingresos a costa de una mayor inflación. Ya en estudios más recientes, Tavits, citado en Pickering \& James (2011) analiza un conjunto de países para los períodos 1974-1983 y 1986-1995, y encuentra que la ideología del partido político esta relacionado con el tamaño del sector público.

Los trabajos posteriores a 1986 ponen énfasis en la contrastación empírica de la Ley de Wagner y no en factores políticos e institucionales. Parece ser que la evidencia valida la ley de Wagner, especialmente para países con menor desarrollo relativo y en menor medida, para países desarrollados en su etapa post industrialización. En este sentido, ha habido un debate intenso sobre si la Ley de Wagner se cumple solo en economías en proceso de industrialización y no en economías avanzadas con un alto grado de desarrollo. Ram, (1987), analiza el cumplimiento de la Ley de Wagner en una muestra de 115 países entre 1950-1980 y 1960-1980, y encuentra evidencia a favor de la Ley de Wagner para el 60\% de países. Santos, 2014, estudia la Ley de Wagner para tres países Africanos, Ghana, Nigeria y Sudáfrica entre 1970 a 2012, a través de análisis de cointegración y mínimos cuadrados ordinarios, aplicado individualmente para cada país y concluye que sí existe causalidad unidireccional que va del ingreso nacional al gasto público (en el sentido de Wagner) únicamente para el caso de Ghana, en tanto que, para Nigeria y Sudáfrica, existe una relación bidirecciónal de causalidad por lo que, no es posible afirmar el cumplimiento de la Ley de Wagner.

La ley de Wagner ha sido sometida a estudio para la economía española por Jaén (2004) entre 1960-1995, encontrándose evidencia favorable. También para la India, Narayan, et.al. (2012), valida la ley de Wagner en Estados Federativos de renta baja, con incremententos en mayor medida del gasto público de consumo que el de capital. Para Italia, Magazzimo (2012) y Cavicchiolia \& Pistoresi (2016) encuentran evidencia parcial de la Ley de Wagner. Para Latinoamérica, la ley de Wagner ha sido sometida a contrastación para las economías de México (Rodriguez, Vanegas-Matínez, \& Lima, 2013) y Colombia por Avella (2009). Ambos estudios afirman que la relación de causalidad va del PIB per cápita al gasto público, es decir, validan la hipótesis de Wagner y no la hipótesis Keynesiana. En la revisión de la literatura no se encontraron trabajos aplicados que aborden las causas del crecimiento del sector público en Ecuador.

Respecto a los datos y metodología utilizados, la mayoría de trabajos sobre el crecimiento del sector público usan series temporales de un determinado país. En este punto, Jaén (2004) menciona que: “aunque 
algunos trabajos primigenios consideraron la posibilidad de contrastación a través de estudios transversales, la mayoría de los autores consideran que estos análisis son inadecuados para un estudio sobre procesos dinámicos como el crecimiento del gobierno"(pp. 14), por lo que el enfoque debería ser individualizado por cada país dada la diferente y compleja realidad económica, social y política que desencadenan los procesos de expansión de la economía pública en cada sociedad.

\section{Materiales y MÉtodos}

A fin de dar respuesta a las preguntas de investigación de este estudio, la metodología a seguir se divide en dos partes:

La primera parte consiste en realizar un análisis de la evolución del tamaño del sector público en Ecuador y una comparativa internacional a fin de situar al país en el contexto de otros países con diferentes niveles de desarrollo; y la segunda, busca analizar y cuantificar la relación entre tamaño del sector público de Ecuador a la luz de las cinco teorías analizadas, utilizando para ello varios modelos econométricos y además, determinar la existencia de una posible relación de causalidad o equilibrio a largo plazo entre crecimiento económico y tamaño del sector público.

\subsection{Datos}

Los datos para realizar la comparativa internacional de la relación entre el tamaño del sector público y PIB per cápita en dólares PPA (Paridad de Poder Adquisitivo) corresponden al año 2015, tomados del programa de comparación internacional del Banco Mundial. Para los datos de la variable del sector público de Ecuador y Argentina, se completó la serie en base a estadísticas del Banco Central del Ecuador y del Instituto Argentino de Análisis Fiscal. En total se tiene una muestra de 82 países cuyo listado se adjunta en el Anexo 1.

Para contrastar los factores asociados al crecimiento del sector público en Ecuador se usan datos que abarcan el período de 1983-2016. La información de las variables incluidas en los modelos econométricos fue tomada de las estadísticas del Banco Central del Ecuador, excepto la variable número de empleados públicos, cuya fuente es el Instituto Ecuatoriano de Seguridad Social IESS para el período 1983-2014 y la Organización Mundial del Trabajo para los años 2015 y 2016.

\subsection{Metodología}

Para la comparativa internacional se aplica el método gráfico y un test de diferencia de medias, con base a la clasificación realizada por el Banco Mundial que agrupa a los países según sus niveles de PIB per cápita en dólares corrientes y que se actualiza cada año conforme se muestra en la tabla 1 (ver listado de países en el anexo 1)

Con ello se busca dar respuesta a la siguiente pregunta de investigación: ¿Está sobredimensionado el tamaño del sector público de Ecuador, en relación a otros países con similares niveles de desarrollo medidos por su PIB per cápita? 
TABLA 1.

Muestra de países según nivel de PIB per cápita: año 2015

\begin{tabular}{l|l|l|l|l|l|l}
\hline \multirow{2}{*}{$\begin{array}{l}\text { Nivel de PIB } \\
\text { perc }\end{array}$} & \multirow{2}{*}{$\begin{array}{l}\text { Rango PIB } \\
\text { en US } \$\end{array}$} & \multirow{2}{*}{$\begin{array}{l}\text { Muestra } \\
\text { (N) }\end{array}$} & \multicolumn{3}{|c}{ Tamaño sector público (\% } \\
\cline { 5 - 7 } & & Media) & $\begin{array}{l}\text { Desviación } \\
\text { estándar }\end{array}$ & Mín & Máx \\
\hline Bajo * & $\begin{array}{l}\text { Menor a } \\
4.125\end{array}$ & 17 & 25,43 & 8,036 & 12 & 41 \\
\hline Medio & $\begin{array}{l}\text { De } 4.126 \text { a } \\
12.735\end{array}$ & 23 & 29,60 & 9,546 & 12 & 50 \\
\hline Alto & $\begin{array}{l}\text { Más de } \\
12.735\end{array}$ & 42 & 39,48 & 10,107 & 15 & 57 \\
\hline Total & & 82 & 33,80 & 11,210 & 12 & 57 \\
\hline
\end{tabular}

Programa de Comparación Internacional del Banco Mundial, Banco Central del Ecuador y del Instituto Argentino de Análisis Fiscal.

*Nota: Países de ingreso bajo el ingreso medio bajo se agrupan en la categoría Bajo a fin de maximizar el tamaño de muestra para este grupo.

Para estimar los modelos econométricos de los factores asociados al crecimiento del sector público en Ecuador, se aplica el método de Mínimos Cuadrados Ordinarios (MCO) y la prueba de raíz unitaria Dickey y Fuller aumentada para determinar la estacionariedad o no estacionariedad de cada variable. Para las regresiones que resulten significativas bajo $\mathrm{MCO}$, se realiza el análisis de cointegración mediante la aplicación del test de Engle y Granger aumentado (EGA) a fin de comprobar que se trata de relaciones de largo plazo entre las dos variables cointregradas y no de una regresión espuria. Adicionalmente, para probar la hipótesis de Wagner, se aplica el test de causalidad de Granger para determinar la relación de causalidad entre el tamaño del Estado y la variable explicativa PIB pér cápita y la dirección de dicha causalidad, a saber: causalidad unidireccional, bidireccional o relación de independencia. Para el análisis se usa el programa estadístico SPSS e Eviews 10.

En la tabla 2 se especifican los modelos para probar empíricamente cada teoría. La variable dependiente que mide el tamaño del sector público en Ecuador es la ratio Gasto Público / PIB denominada GOV y en logaritmos LN_GOV. El gasto público abarca las operaciones del sector público no financiero que incluye los gastos corrientes y de capital de las empresas públicas no financieras, de los gobiernos seccionales y del gobierno central. Se excluye el gasto en intereses de la deuda, a fin de reflejar el consumo real en bienes y servicios, personal, inversiones y de capital del sector público dentro de la economía.

TABLA 2.

Resumen de modelos econométricos planteados para estimar los factores asociados al crecimiento del sector público en Ecuador

\begin{tabular}{|c|c|c|}
\hline Teoria Explicativa & Especificación econométrica & $\begin{array}{l}\text { Signo esperado del } \\
\text { coeficiente }\end{array}$ \\
\hline \multirow{2}{*}{ Ley de Wagner. } & 1. & $\beta 1>0$ \\
\hline & 2. & $\beta 1>0$ \\
\hline Internacionalización. & 3. & $\beta 1>0$ \\
\hline Burocracia. & 4. & $\beta 1>0$ \\
\hline \multirow{3}{*}{ Ilusión Fiscal. } & 5 & $\beta 1>0$ \\
\hline & 6 & $\beta 1>0$ \\
\hline & 7. & $\beta 1>0$ \\
\hline \multirow{2}{*}{$\begin{array}{l}\text { Ideología del Partido } \\
\text { Político. }\end{array}$} & 8. & $\beta 1>0$ \\
\hline & 9. & $\mathrm{~B} 3>0$ \\
\hline
\end{tabular}


El autor.

En la tabla 3, se presenta la definición de las variables independientes para cada una de las teorías explicativas y el signo teórico esperado de los coeficientes, que para las relaciones planteadas en este estudio, se espera que sean todos positivos.

TABLA 3.

Definición de las variables explicativas usadas en el estudio

\begin{tabular}{|c|c|c|c|}
\hline Teoría Explicativa & $\begin{array}{l}\text { Nombre de la } \\
\text { Variable } \\
\text { explicativa }\end{array}$ & Descripción de la Variable & $\begin{array}{l}\text { Signo } \\
\text { teórico } \\
\text { esperado }\end{array}$ \\
\hline \multirow[b]{2}{*}{ 1. Ley de Wagner } & LN PIB perc & $\begin{array}{l}\text { Logaritmo natural del PIB per cápita real } \\
\text { en dólares del } 2007\end{array}$ & + \\
\hline & Grado_urb & $\begin{array}{l}\text { Grado de urbanización: Porcentaje de la } \\
\text { población urbana / total de la población } \\
\text { del pais }\end{array}$ & + \\
\hline $\begin{array}{l}2 . \\
\text { Internacionalización }\end{array}$ & Apertura & $\begin{array}{l}\text { Logaritmo natural del Grado de apertura } \\
\text { de la economia: LN (Exportaciones }+ \\
\text { Importaciones)/PIB }\end{array}$ & + \\
\hline 3. Burocracia & Emp_público & $\begin{array}{l}\text { Logaritmo natural (número de } \\
\text { empleados públicos/ total de la } \\
\text { población del pais) }\end{array}$ & + \\
\hline \multirow{3}{*}{ 4. Ilusión Fiscal } & Prec_WTI & $\begin{array}{l}\text { Logaritmo natural del precio x barril de } \\
\text { petróleo WTI }\end{array}$ & + \\
\hline & Imp_ind t-1 & $\begin{array}{l}\text { Logaritmo natural de ingresos x } \\
\text { impuestos indirectos / Ingresos totales } \\
\text { del Gobierno Central del periodo t-1. } \\
\text { (Impuestos indirectos incluyen IVA, ICE } \\
\text { e impuestos arancelarios) }\end{array}$ & + \\
\hline & Deuda t-1 & $\begin{array}{l}\text { Logaritmo natural del saldo de la deuda } \\
\text { pública al final de cada año / PIB } \\
\text { periodo t-1 }\end{array}$ & + \\
\hline \multirow[b]{2}{*}{$\begin{array}{l}\text { 5. Ideología del } \\
\text { Partido Político }\end{array}$} & D1 & $\begin{array}{l}\text { Variable binaria con dos categoría: } \mathrm{D}= \\
1 \text { (si el partido es de izquierda); } 0 \text { (si el } \\
\text { partido es de derecha) }\end{array}$ & + \\
\hline & $\mathrm{D} 1 ; \mathrm{D} 2 ; \mathrm{D} 3$ & $\begin{array}{l}\text { Variable binaria con } 3 \text { categorias: } \\
\text { Categoria base: } 0 \text { si el partido es de } \\
\text { derecha. D } 1:=1 \text { si el partido es de } \\
\text { centro derecha; } 0 \text { para todo lo demás. } \\
\text { D2 = } 1 \text { si el partido es de centro } \\
\text { izquierda; } 0 \text { para todo lo demás; } \mathrm{D} 3=1 \\
\text { si el partido es de izquierda; } 0 \text { para todo } \\
\text { lo demás. }\end{array}$ & + \\
\hline
\end{tabular}

$\mathrm{Ui}=$ Término de error

\section{Resultados y Discusión}

\subsection{Tamaño del sector público en Ecuador desde una comparativa internacional}

En la muestra de 82 países presentada en el gráfico 1, la relación entre tamaño del sector público y PIB per cápita en PPA sigue una trayectoria de una U invertida (ajuste cuadrático con bondad de ajuste de 0,32), en donde a medida que se incrementa el PIB per cápita, el tamaño del sector público también crece, hasta llegar a un punto de inflexión correspondiente a un nivel de renta de entre los US\$50.000 y US\$ 60.000, a partir del cual, la relación entre estas variables es negativa. Esta relación es denominada en la literatura económica como curva de Armey y puede interpretarse como una prolongación de la curva de Laffer que expresa la relación entre nivel impositivo y crecimiento económico. Asimakopoulos \& Karavias (2016) en su estudio sobre el 
impacto del tamaño del gobierno en el crecimiento económico para 109 países en el período 1980 - 2009, encuentran que existe una relación no lineal entre tamaño del estado y crecimiento económico en forma de U invertida.

La interpretación más intuitiva de la curva de Armey (gráfico 1), dice que en un primer momento, el crecimiento del sector público genera efectos positivos en el PIB per cápita, pero a medida que el sector público crece, llega un momento (punto de inflexión) en que por su tamaño se convierte en una pesada carga para los contribuyentes, llegando a superar la capacidad de la economía para sostenerlo. En este punto de inflexión, cuando el PIB per cápita ha alcanzado su máximo, la productividad marginal del gasto público es igual a la productividad marginal del gasto privado (Altunc \& Aydin, 2013), por lo que desde este nivel, la contribución económica del sector público se vuelve cero y no genera efectos positivos en el crecimiento económico, sino su estancamiento. Para interpretar el gráfico 1 , se procedió a dividirlo en cuatro cuadrantes en función de la mediana del tamaño del sector público (33,7\% respecto del PIB) y la mediana del PIB per cápita (25.048 US\$).

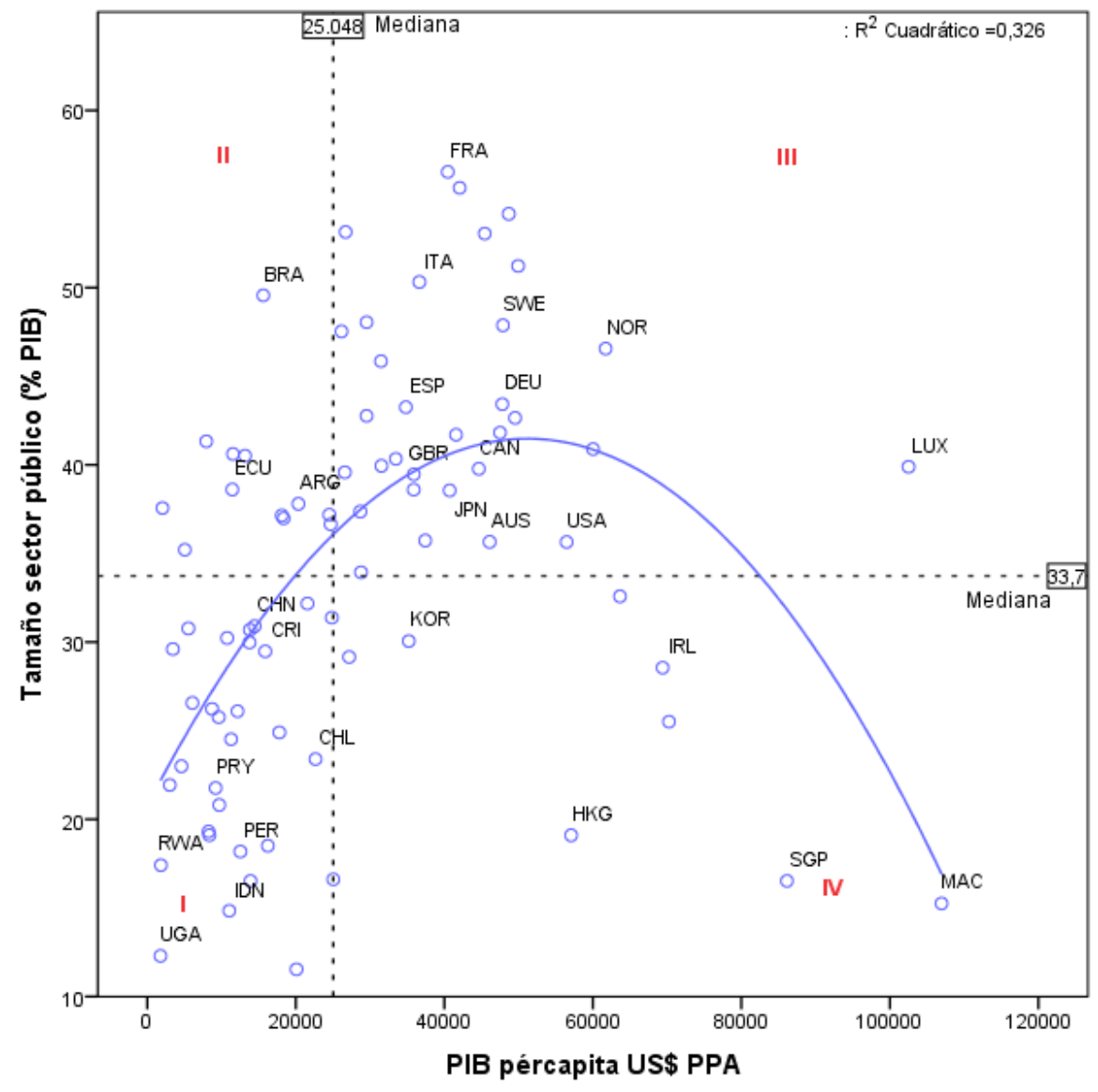

GRÁFICO 1.

Tamaño del sector público y PIB per cápita US\$ PPA.

Comparación internacional (muestra de 82 países) año 2015

Datos del Banco Mundial, Instituto Argentino de Análisis Fiscal y Banco Central del Ecuador.

El cuadrante I, agrupa a países con PIB per cápita y tamaño del sector público inferior a la mediana, en donde encontramos países de ingreso medio de la región latinoamericana como Perú (PER), Colombia (COL), Costa Rica (CRI), Chile (CHL) y Paraguay (PRY), así como China (CHN) en Asia, y países de ingreso bajo de África como Ruanda (RWA) y Uganda (UGA). En el segundo cuadrante se agrupan países con un sector público grande superior a $33,7 \%$ pero con un desarrollo económico medido por su PIB per 
cápita similar a los países del cuadrante 1. Para Latinoamérica encontraríamos en esta posición a países como Brasil (BRA), Ecuador (ECU), Argentina (ARG), los cuales, a pesar de destinar una gran proporción de recursos de la economía para financiar las actividades estatales, no han conseguido diferenciarse en cuanto a resultados económicos de los países del cuadrante uno que con un menor tamaño de su sector público logran similares niveles de ingreso per cápita. Si miramos por el tamaño del sector público, estos países del cuadrante II, tienden a parecerse a países desarrollados (cuadrante III) como España (ESP), Reino Unido (GBR), o Alemania (DEU), pero muy por debajo en cuanto a niveles de ingreso per cápita.

\subsection{Comparativa del tamaño del sector público de Ecuador con paises de similares niveles de PIB per cápita}

A continuación, a fin de determinar si la evidencia estadística apoya las conclusiones del análisis gráfico, se aplica un test de diferencia de medias (tabla 4) entre el tamaño del sector público de Ecuador y el grupo de países de renta media, medido por su PIB per cápita en PPA.

La hipótesis nula $(\mathrm{Ho})$ a contrastar es que la media del tamaño del sector público de Ecuador y del grupo de países de renta media es la misma, es decir no existe diferencia estadística significativa.

TABLA 4.

Resultados: Test de diferencia de medias entre tamaño del sector público (\% PIB) de Ecuador vs países de nivel medio de PIB per cápita. Datos para el año 2015

\begin{tabular}{|c|c|c|c|c|c|c|c|c|}
\hline \multicolumn{2}{|c|}{$\begin{array}{l}\text { Tamaño sector público como \% del } \\
\text { PIB }\end{array}$} & \multirow[b]{2}{*}{ Muestra (N) } & \multirow[b]{2}{*}{ Desviación estándar } & \multirow[b]{2}{*}{ Diferencia de Medias } & \multirow[b]{2}{*}{$\mathrm{t}$} & \multirow[b]{2}{*}{ gl } & \multirow{2}{*}{\multicolumn{2}{|c|}{ Sig. }} \\
\hline $\begin{array}{l}\text { Países nivel } \\
\text { Medio de PIB per } \\
\text { cápita }\end{array}$ & $\begin{array}{l}\text { Valor de Prueba } \\
\text { (Ecuador) }\end{array}$ & & & & & & & \\
\hline 29,60 & 37,24 & 23 & 9,546 & $-7,64$ & $-3,830$ & 22 & .001 & \\
\hline
\end{tabular}

Datos del Banco Mundial, Instituto Argentino de Análisis Fiscal y Banco Central del Ecuador.

Según estos resultados, no existe evidencia suficiente para aceptar la hipótesis nula de igualdad de medias; se concluye que el tamaño del sector público de Ecuador está sobredimensionado en un 7,64\% en comparación con el promedio del sector público $(29,60 \%)$ de países con similares niveles de renta per cápita en US\$ PPA al año 2015.

\subsection{Factores asociados al crecimiento del sector público}

Los resultados de las especificaciones para estimar los factores asociados al crecimiento de la economía pública en Ecuador se presentan en la tabla 5. 
TABLA 5.

Resultados: Factores asociados a la expansión del tamaño del sector público en Ecuador en el período 1983-2016

\begin{tabular}{|c|c|c|c|c|c|c|c|}
\hline Teoría Explicativa & $\begin{array}{l}\text { Variable } \\
\text { Dependiente }\end{array}$ & $\begin{array}{l}\text { Variable } \\
\text { Independiente }\end{array}$ & $\begin{array}{l}\text { Signo } \\
\text { teórico } \\
\text { esperado }\end{array}$ & $\begin{array}{l}\text { Signo } \\
\text { encontrado } \\
\text { y } \\
\text { Significancia } \\
\text { estadistica }\end{array}$ & $\begin{array}{l}\text { Valor } \\
\text { coeficiente } \\
(\beta)\end{array}$ & $\begin{array}{l}\text { Correlación } \\
\text { Simple }\end{array}$ & $\mathrm{R} 2$ \\
\hline \multirow{2}{*}{ 1. Ley de Wagner } & LN GOV & LN PIB perc & + & $+* * *$ & 1,33 & 0,68 & 0,47 \\
\hline & GOV & Grado_urb & + & $+* * *$ & 1,13 & 0,50 & 0,32 \\
\hline \multirow{2}{*}{$\begin{array}{l}\text { 2. } \\
\text { Internacionalización } \\
\text { 3. Burocracia }\end{array}$} & LN GOV & Apertura & + & $+^{* * *}$ & 0,573 & 0,38 & 0,15 \\
\hline & $\begin{array}{l}\text { LN GOV } \\
\text { LN GOV }\end{array}$ & $\begin{array}{l}\text { Emp_público } \\
\text { Prec_WTI }\end{array}$ & $\begin{array}{l}+ \\
+\end{array}$ & $\begin{array}{l}+^{* * *} \\
t^{* * *}\end{array}$ & $\begin{array}{l}0,868 \\
0,19\end{array}$ & $\begin{array}{l}0,762 \\
0,53\end{array}$ & $\begin{array}{l}0,58 \\
0,29\end{array}$ \\
\hline \multirow[t]{3}{*}{ 4. Ilusión Fiscal } & LN GOV & $\operatorname{Imp}$ Ind $t-1$ & + & $-N$ & $-0,14$ & $-0,12$ & 0,01 \\
\hline & LN GOV & Deuda t-1 & + & $-* *$ & $\begin{array}{l}-0,12 \\
\beta 0=\end{array}$ & $-0,49$ & 0,49 \\
\hline & GOV & Intercepto D1 & + & $+* * *$ & $\begin{array}{l}0,20 \\
\beta 1 \quad 0,118\end{array}$ & & 0,69 \\
\hline \multirow[t]{2}{*}{$\begin{array}{l}\text { 5. Ideología del } \\
\text { Partido Político }\end{array}$} & GOV & $\begin{array}{l}\text { Intercepto D1 } \\
\text { (Centro } \\
\text { Derecha) }\end{array}$ & - & $-N$ & $\begin{array}{l}\beta 0= \\
0,21 \\
\beta 1=- \\
0,035\end{array}$ & & 0,87 \\
\hline & & $\begin{array}{l}\text { D2 (Centro } \\
\text { Izquierda) } \\
\text { D3 ( } \\
\text { Izquierda) }\end{array}$ & $\begin{array}{l}+ \\
+ \\
\end{array}$ & $\begin{array}{r}N \\
+* * * \\
\end{array}$ & $\begin{array}{l}\beta 2=0,00 \\
\beta 3=0,15\end{array}$ & & \\
\hline
\end{tabular}

Elaboración propia.

Debido a que los resultados anteriores están soportados por datos de series temporales, a las regresiones que resultaron significativas, bajo el método de mínimos cuadrados ordinarios, se les aplicó el análisis de cointegración mediante el test de Engle y Granger aumentado (EGA) cuyos resultados se muestran en la tabla 6 , a fin de comprobar que se trata de relaciones de largo plazo entre las dos variables cointregradas.

TABLA 6.

Relación de cointegración Engle-Granger entre el tamaño del sector público y las variables explicativas significativas en los modelos de MCO

\begin{tabular}{|c|c|c|c|c|c|c|}
\hline Teoría Explicativa & $\begin{array}{l}\text { Variable } \\
\text { Independiente }\end{array}$ & $\begin{array}{l}\text { Hipótesis nula } \\
\text { (Ho) }\end{array}$ & Estadístico ct & $\begin{array}{l}\text { Valor crítico } \\
(5 \%)\end{array}$ & $\begin{array}{l}\text { Valor crítico } \\
(10 \%)\end{array}$ & Resultado \\
\hline \multirow{2}{*}{ Ley de Wagner } & LN PIB perc & Tiene raiz unitaria & $-3,25$ & $-3,5$ & $-3,04$ & $*$ \\
\hline & Grado_urb & Tiene raiz unitaria & $-3,59$ & $-3,5$ & $-3,04$ & $* *$ \\
\hline Internacionalización & Grado de Apertura & Tiene raiz unitaria & $-3,18$ & $-3,50$ & $-3,04$ & * \\
\hline Burocracia & Emp_público & Tiene raiz unitaria & $-3,55$ & $-3,5$ & $-3,04$ & $* *$ \\
\hline Ilusión Fiscal & Prec_WTI & Tiene raiz unitaria & $-2,01$ & $-3,5$ & $-3,04$ & Acepta Ho \\
\hline
\end{tabular}


De las cinco teorías explicativas del crecimiento del sector público en Ecuador, únicamente para dos de estas teorías (ideología del partido político y burocracia) se encontró sustento empírico fuerte. A continuación, se analizan los resultados obtenidos:

\section{Ley de Wagner}

Los coeficientes estimados de la ley de Wagner, resultaron con el signo teórico esperado y con significancia estadística a un nivel del 0,1\% de confianza (Tabla 5). El grado de urbanización afecta de forma positiva al crecimiento del sector público y es significativo. El coeficiente 1,33 que mide la elasticidad del tamaño del sector público respecto del PIB per cápita es significativo y mayor a 1 , lo cual indicaría que la demanda por bienes y servicios públicos es elástica con respecto a la renta, como lo predice la teoría; esta alta elasticidad significa que la demanda por dichos bienes, por parte de los individuos, aumenta en forma más que proporcional al aumento del ingreso per cápita, lo que conlleva al crecimiento del tamaño del sector público respecto al total de la economía. El análisis de cointegracion de Engle y Granger (Tabla 6) para la primera especificación de la ley de Wagner, permite aceptar la hipótesis alternativa de residuos estacionarios (no tienen raíz unitaria) y por tanto, existe una relación de equilibrio de largo plazo entre el PIB per cápita y el tamaño del sector público únicamente para un nivel de significancia del $10 \%$. Con respecto a la segunda especificación, a un nivel de confianza del $5 \%$ no hay evidencia suficiente que permita aceptar la hipótesis nula de no estacionariedad y por tanto, existe una relación de largo plazo entre el grado de urbanización del país y el tamaño del sector público. Con estos resultados, podemos afirmar que se encontró evidencia parcial a favor de la ley de Wagner como uno de los factores que ha impulsado la expansión del sector público de Ecuador entre 1983-2016. Adicionalmente, se procedió a realizar un análisis de causalidad de Granger entre el tamaño del sector público y el crecimiento del PIB per cápita y explorar su implicancia en términos de política económica (Tabla 7). De acuerdo con los resultados de la prueba de causalidad, se descarta la posibilidad de que sea el gasto público el que determina la actividad económica medida por el PIB per cápita, es decir de que la causalidad sea en el sentido (LN GOV $\rightarrow$ LN PIB per), lo cual va en contra de la hipótesis keynesiana. Por el contrario, a un nivel de confianza del 10\%, se acepta la hipótesis alternativa de que la relación de causalidad sea (LN PIB per $\rightarrow$ LN GOV).

TABLA 7.

Prueba de causalidad de Granger

\begin{tabular}{|c|c|c|c|}
\hline Hipótesis nula & $\begin{array}{l}\text { Relación de } \\
\text { Causalidad en el } \\
\text { Largo Plazo }\end{array}$ & Chi-sq & Prob. \\
\hline $\begin{array}{l}\text { LN PIB perc causa a } \\
\text { LN GOV } \\
\text { LN GOV causa a LN } \\
\text { PIB perc }\end{array}$ & $\begin{array}{l}\text { LN PIB per } \rightarrow \text { LN } \\
\text { GOV } \\
\text { LN GOV } \rightarrow \text { LN } \\
\text { PIB per }\end{array}$ & $\begin{array}{l}5.392581 \\
0.385480\end{array}$ & $\begin{array}{l}0.0675 \\
0.8247\end{array}$ \\
\hline
\end{tabular}

Elaboración propia.

Ampliando el análisis de estos resultados, habría que considerar que el crecimiento del sector público respecto del total de la economía ecuatoriana no se debe únicamente a factores económicos (demandas sociales derivadas del progreso de la sociedad) que se sintetizan en la explicación de Wagner. Las bajas tasas de crecimiento económico que ha experimentado el país (ingreso per cápita real creció a un promedio anual de 1,13\%) entre 1983 - 2016, si se las compara con el crecimiento del sector público (relación sector público / PIB creció a una tasa del 4,63\%), permite prever que hay otros elementos a más de los puramente económicos en la expansión del tamaño del gobierno que juegan un rol preponderante. Wildavsk citado en (Cameron, 1978), argumenta que el sector público se expande de forma creciente en términos relativos, cuando la tasa de crecimiento económico es modesta, ya que, en naciones de alto crecimiento, los fondos para financiar las actividades estatales pueden satisfacerse más fácilmente con el "dividendo" resultante de aplicar 
una proporción constante (gasto público / PIB) a un producto económico en expansión. Por el contrario, en naciones con bajo crecimiento económico, los gobiernos no disfrutan de ese dividendo, y en consecuencia, los fondos requeridos por el estado absorben cada vez una mayor parte del producto de la economía.

\section{Internacionalización}

El valor del coeficiente de elasticidad entre el tamaño del sector público y el grado de apertura igual a 0,573 , resultó significativo a un nivel de confianza del $5 \%$ y con el signo predicho por la teoría de la internacionalización. Sin embargo, bajo el análisis de cointegración únicamente fue posible aceptar la hipótesis de que la relación entre estas dos variables no se trata de una correlación espuria a un nivel de confianza del $10 \%$. Por tanto, no se encontró evidencia robusta en apoyo a la tesis de que la internacionalización económica, medida como el grado de apertura comercial, es un factor asociado al crecimiento del sector público en Ecuador. Otros autores como Jetter \& Parmeter (2014) que estudian la relación entre apertura comercial y tamaño del sector público para una muestra de 154 países para el período 1960-2000, han encontrado evidencia de que en promedio, un mayor grado de apertura comercial repercute en un mayor tamaño del sector público.

\section{Burocracia}

La influencia y el poder del voto burocrático en el sistema democrático y en las políticas públicas resultó tener sustento empírico para el caso ecuatoriano. El coeficiente de elasticidad 0,86 resultó estadísticamente significativo y con el signo esperado; la bondad de ajuste medida por el coeficiente de determinación $R^{2}$ es de 0,58 y la correlación entre ambas variables es igual a 0,76 . El coeficiente 0,86 se puede interpretar de la siguiente forma: a medida que el número de empleados públicos con respeto a la población total del país se incrementa en un 1\%, el tamaño del sector público (gasto público / PIB) se incrementa en una proporción estimada del $0,86 \%$. El análisis de cointegración, refuerza estos resultados y revela que existe una relación de largo plazo entre el tamaño del sector público y el número de empleados públicos como proporción de la población total del país, ya que el valor del estadístico del test de Engle y Granger de $-3,55$, menor al valor crítico de -3,5, no permite aceptar la hipótesis nula de no cointegración (presencia de raíz unitaria).

Si bien la evidencia encontrada nos permite concluir que la burocracia tiene implicaciones importantes en la expansión del gasto público, el hecho de que el coeficiente de elasticidad sea menor a uno, no permite afirmar que este efecto sea en el sentido propuesto por Buchanan y Tullock como Ley de Wagner al cuadrado, en donde se argumentan que el sector público estaría fuera de control debido al poder político de los funcionarios que presionan al alza el gasto público en general y los salarios en particular, en contraposición a los intereses de los votantes. El efecto de la burocracia, no solo depende del número de empleados públicos, sino también de elementos institucionales de la misma estructura del Estado tales como el grado de discrecionalidad y concentración de poder especialmente en el poder ejecutivo y cargos intermedios encargados de la ejecución de gastos. En el caso de Ecuador, con la aprobación de la Constitución del 2008 y el ordenamiento jurídico derivado de este proceso, parecería haberse transitado a un modelo de mayor discrecionalidad y concentración de poder en el ejecutivo que favorece en mayor medida la expansión del gasto público.

\section{Ilusión Fiscal}

De las tres variables explicativas usadas para medir el efecto de la ilusión fiscal como fenómeno explicativo del gasto público en Ecuador, solo la variable precios del petróleo resultó ser significativa y tener el signo esperado. Sin embargo, el análisis de cointegración revela que a un nivel del 5\% y del 10\% no es posible aceptar la hipótesis alternativa de estacionariedad, por lo que no existiría una relación de largo plazo entre estas variables.

Para el caso de la variable impuestos indirectos, el coeficiente es negativo contrario al predicho por aquellos autores que defienden este argumento, aunque su valor no es estadísticamente diferente de cero. Basados en esta evidencia, podemos deducir que, la estructura impositiva del país, en donde al año 2016 los impuestos indirectos representaron el 25,8\% de los ingresos totales del Estado, no tiene incidencia en el crecimiento 
del sector público. Otros autores han encontrado evidencia de que la ilusión fiscal creada por el sistema impositivo no tiene efecto sobre la expansión de la economía pública. Cameron (1978), en su estudio del crecimiento del sector público en 18 naciones en 1960, encontró que los presupuestos no se expanden más rápidamente cuando los impuestos se ocultan bajo el fenómeno de "ilusión fiscal" y por el contrario, una dependencia creciente de los impuestos "ocultos" ejerció un efecto atenuador significativo sobre el ritmo de expansión de la actividad estatal, hallando una fuerte relación negativa entre estas variables.

\section{Ideología del partido político}

Los resultados obtenidos introduciendo como categoría de análisis la ideología del partido político proveen evidencia, a favor de la tesis de que el crecimiento del sector público en Ecuador está estrechamente relacionado a factores políticos y más específicamente a la ideología del partido político, que ostenta el poder dentro de su sistema democrático. El tamaño promedio del sector público (Gasto público / PIB) en períodos en el que el poder ejecutivo estuvo en manos de gobiernos de derecha medido en el modelo por el coeficiente fue del $20 \%$. El coeficiente $\beta 1$ estimado que mide la diferencia promedio del tamaño del sector público en gobiernos de izquierda, en comparación con un gobierno de derecha, es estadísticamente diferente de cero a un $1 \%$ de nivel de confianza. Es decir, en períodos de gobierno de izquierda el tamaño promedio del sector público es de $(\beta 0+\beta 1=31,8 \%)$, lo cual es superior en un $11,8 \%$ a un gobierno de derecha.

\section{Conclusiones}

De las cinco posibles teorías explicativas del crecimiento del sector público en Ecuador, los resultados revelan evidencia empírica fuerte a favor de que el crecimiento del sector público está asociado a factores de orden político como la ideología del partido de gobierno e institucional, identificados como los efectos de la burocracia sobre el sistema democrático. Se encontró evidencia parcial a favor de la Ley de Wagner y la tesis de la internacionalización de la economía, y ningún soporte en apoyo a la ilusión fiscal creada por el sistema impositivo o por el efecto distorsionador de los ingresos fiscales provenientes de un recurso natural como el petróleo o la financiación mediante deuda pública.

Respecto de la ideología del partido político, los resultados muestran que el tamaño del sector público en Ecuador, en períodos de gobiernos de izquierda, es superior en un $11,8 \%$ si se lo compara con los gobiernos de derecha. Efectivamente, a partir del año 2008 con la aprobación de un nuevo marco constitucional y la entrada en la escena política ecuatoriana de un nuevo partido de gobierno, el tamaño del sector público se elevó de forma considerable.

Otro factor determinante, asociado al tamaño del sector público en Ecuador, es la influencia de la burocracia. El análisis de cointegración revela que existe una correlación positiva y una relación de largo plazo entre el tamaño del sector público y el número de empleados públicos, como proporción de la población total, cuya elasticidad se estima en 0,86 .

Se halló evidencia parcial a favor de la existencia de una relación de equilibrio a largo plazo entre crecimiento económico y tamaño del sector público, por lo que se exploró la posible relación entre estas dos variables mediante el test de causalidad de Granger. De los resultados obtenidos, se descarta la posibilidad de que sea el gasto público el que determina la actividad económica, es decir la relación de causalidad va del PIB per cápita al gasto público (LN PIB per $\rightarrow$ LN GOV) y no al revés para el caso de la Economía de Ecuador. Esta evidencia tiene importantes implicaciones de política económica debido a que contradice la teoría keynesiana ya que, si la causalidad va del ingreso al gasto público, este último no puede ser usado como un instrumento exógeno para incidir en el crecimiento económico y en el ingreso agregado en el largo plazo.

El análisis comparativo a nivel internacional muestra que no existe una relación directa entre prosperidad económica medida por el PIB per cápita y tamaño del sector público. Muchos de los países con mayor ingreso per cápita tienen un sector público que absorbe más del 40\% del PIB sobre todo en Europa, sin embargo, también existen países con un sector público relativamente pequeño con altos estándares de vida. 
En el caso de Ecuador, según datos del Banco Central del Ecuador, el tamaño del sector público que al año 2015 llegó al 37,24\% del PIB es muy superior al de países con similar nivel de ingresos per cápita cuyo promedio para ese mismo año se estimó en 29,60\%, característica que lo acerca más a economías desarrolladas, pero sin los mismos resultados en cuanto a estándares de vida y nivel de ingresos. Autores como Asimakopoulos \& Karavias, (2016) que analizan el impacto del tamaño del estado en el crecimiento económico para una muestra de 129 países, encuentran que hay un impacto asimétrico del tamaño de gobierno sobre el crecimiento económico en países del mundo desarrollado y en desarrollo. Para países en desarrollo, el efecto negativo de un tamaño del estado por encima del nivel óptimo en la curva de Armey es más significativo que el efecto positivo de un tamaño del estado por debajo del óptimo. En cambio, los países desarrollados tienen fuertes efectos positivos cuando se incrementa el tamaño del estado desde un nivel por debajo del límite óptimo en comparación con los efectos negativos cuando este sobrepasa el óptimo estimado. "Este resultado podría provenir del hecho de que los países desarrollados tienen mejor calidad de instituciones gubernamentales para explotar todos los beneficios derivados de un mayor gasto público y mitigar sus efectos negativos" (Asimakopoulos \& Karavias, 2016).

Por tanto, ante un sector público excesivo, resulta evidente que en Ecuador se tendría que tender a su reducción, a fin de recuperar la senda del crecimiento, buscando minimizar los costos sociales derivados de este proceso, ya que, a partir de un determinado nivel, un mayor crecimiento del sector público significa estancamiento económico y excesiva carga fiscal sobre los contribuyentes.

El factor político también juega un importante rol en la efectividad del gasto público; los esfuerzos de un determinado gobierno que aspire aumentar el gasto público para solucionar los problemas sociales más apremiantes, se verán reducidos de forma significativa debido a la propia institucionalidad pública que favorece la aparición de grupos de interés con un comportamiento capturador de rentas. Este hecho tiene importantes repercusiones en materia redistributiva y podría incluso resultar regresivo en la medida en que los grupos de interés, no necesariamente, representen a los individuos con menor nivel de renta que por lo general tienen menor poder e influencia política.

Estos resultados cuestionan la idea enraizada en los últimos años, en gran parte de la sociedad ecuatoriana y en algunos países de la región, de que el crecimiento económico, el progreso, la equidad y la cohesión social dependen en gran medida del fortalecimiento, la presencia e intervención del sector público, no solo en la economía, sino también, en varios otros aspectos de la vida social, lo cual resulta ser muy atractivo para los políticos que operan en un sistema democrático con baja calidad institucional. 
Juan B. Matute Pacheco. Factores explicativos del crecimiento del sector público. El caso de Ecuad...

\section{ANEXo 1:}

\begin{tabular}{|c|c|c|c|c|c|c|}
\hline Año & País & & Región & $\begin{array}{c}\text { PIB PPA en } \\
\text { USS }\end{array}$ & $\begin{array}{c}{ }^{*} \mathrm{SP} / \mathrm{PIB} \\
(\%)\end{array}$ & $\begin{array}{c}\text { Nivel de } \\
\text { Ingreso en } \\
\text { PPA }\end{array}$ \\
\hline 2015 & Armenia & ARM & Europa y Asia central & 8.728 & 26,23 & Bajo \\
\hline 2015 & Bhután & BTN & Asia meridional & 8.246 & 19,31 & Bajo \\
\hline 2015 & Egipto, República Árabe de & EGY & Oriente Medio y Norte de África & 10.750 & 30,23 & Bajo \\
\hline 2015 & El Salvador & SLV & América Latina y el Caribe & 8.354 & 19,11 & Bajo \\
\hline 2015 & Honduras & HND & América Latina y el Caribe & 4.591 & 22,99 & Bajo \\
\hline 2015 & Indonesia & IDN & Asia oriental y el Pacifico & 11.040 & 14,84 & Bajo \\
\hline 2015 & Kenya & KEN & África al sur del Sahara & 3.020 & 21,93 & Bajo \\
\hline 2015 & Kirguistán & $\mathrm{KGZ}$ & Europa y Asia central & 3.448 & 29,60 & Bajo \\
\hline 2015 & Kosovo & $\mathrm{XKX}$ & Europa y Asia central & 9.687 & 20,81 & Bajo \\
\hline 2015 & Mongolia & MNG & Asia oriental y el Pacífico & 12.152 & 26,10 & Bajo \\
\hline 2015 & República de Moldova & MDA & Europa y Asia central & 5.055 & 35,21 & Bajo \\
\hline 2015 & Rwanda & RWA & África al sur del Sahara & 1.827 & 17,41 & Bajo \\
\hline 2015 & Tonga & TON & Asia oriental y el Pacífico & 5.526 & 30,77 & Bajo \\
\hline 2015 & Timor-Leste & TLS & Asia oriental y el Pacífico & 2.045 & 37,57 & Bajo \\
\hline 2015 & Ucrania & UKR & Europa y Asia central & 7.949 & 41,33 & Bajo \\
\hline 2015 & Uganda & UGA & África al sur del Sahara & 1.774 & 12,29 & Bajo \\
\hline 2015 & Uzbekistán & UZB & Europa y Asia central & 6.070 & 26,57 & Bajo \\
\hline 2015 & Albania & ALB & Europa y Asia central & 11.283 & 24,52 & Medio \\
\hline 2015 & Argentina & ARG & América Latina y el Caribe & 20.340 & 37,80 & Medio \\
\hline 2015 & Azerbaiyán & AZE & Europa y Asia central & 17.782 & 24,90 & Medio \\
\hline 2015 & Belarús & BLR & Europa y Asia central & 18.347 & 36,98 & Medio \\
\hline 2015 & Bosnia y Herzegovina & $\mathrm{BIH}$ & Europa y Asia central & 11.526 & 40,61 & Medio \\
\hline 2015 & Brasil & BRA & América Latina y el Caribe & 15.617 & 49,56 & Medio \\
\hline 2015 & Bulgaria & BGR & Europa y Asia central & 18.115 & 37,15 & Medio \\
\hline 2015 & China & CHN & Asia oriental y el Pacífico & 14.450 & 30,90 & Medio \\
\hline 2015 & Colombia & $\mathrm{COL}$ & América Latina y el Caribe & 13.828 & 30,69 & Medio \\
\hline 2015 & Costa Rica & CRI & América Latina y el Caribe & 15.882 & 29,48 & Medio \\
\hline 2015 & Ecuador & $\mathrm{ECU}$ & América Latina y el Caribe & 11.446 & 38,61 & Medio \\
\hline 2015 & Ex República Yugoslava de & MKD & Europa y Asia central & 13.766 & 29,98 & Medio \\
\hline 2015 & Rusia & RUS & Europa y Asia central & 24.692 & 36,65 & Medio \\
\hline 2015 & Georgia & GEO & Europa y Asia central & 9.611 & 25,76 & Medio \\
\hline 2015 & Kazajstán & KAZ & Europa y Asia central & 25.048 & 16,61 & Medio \\
\hline 2015 & Mauricio & MUS & África al sur del Sahara & 20.088 & 11,54 & Medio \\
\hline 2015 & Paraguay & PRY & América Latina y el Caribe & 9.200 & 21,77 & Medio \\
\hline 2015 & Perú & PER & América Latina y el Caribe & 12.531 & 18,18 & Medio \\
\hline 2015 & Rumania & $\mathrm{ROU}$ & Europa y Asia central & 21.566 & 32,17 & Medio \\
\hline 2015 & Serbia & SRB & Europa y Asia central & 13.896 & 16,52 & Medio \\
\hline 2015 & Sudáfrica & $\mathrm{ZAF}$ & África al sur del Sahara & 13.164 & 40,52 & Medio \\
\hline 2015 & Tailandia & THA & Asia oriental y el Pacífico & 16.225 & 18,51 & Medio \\
\hline 2015 & Turquía & TUR & Europa y Asia central & 24.845 & 31,38 & Medio \\
\hline
\end{tabular}

*SP / PIB = Tamaño del Sector Público / PIB

Países incluidos en el análisis de comparación internacional, clasificados por nivel de ingreso en US\$ PPA 


\begin{tabular}{|c|c|c|c|c|c|c|}
\hline Año & País & & Región & $\begin{array}{c}\text { PIB PPA en } \\
\text { US\$ }\end{array}$ & $\begin{array}{c}\text { *SP / PIB } \\
(\%)\end{array}$ & $\begin{array}{c}\text { Nivel de } \\
\text { Ingreso en } \\
\text { PPA }\end{array}$ \\
\hline 2015 & Alemania & DEU & Europa y Asia central & 47.811 & 43,42 & Alto \\
\hline 2015 & Australia & AUS & Australia & 46.095 & 35,66 & Alto \\
\hline 2015 & Austria & AUT & Europa y Asia central & 49.938 & 51,23 & Alto \\
\hline 2015 & Bélgica & BEL & Europa y Asia central & 45.431 & 53,05 & Alto \\
\hline 2015 & Canadá & CAN & Norteamérica & 44.647 & 39,78 & Alto \\
\hline 2015 & Chile & $\mathrm{CHL}$ & América Latina y el Caribe & 22.647 & 23,40 & Alto \\
\hline 2015 & Chipre & CYP & Europa y Asia central & 31.524 & 39,95 & Alto \\
\hline 2015 & Corea, República de & KOR & Asia oriental y el Pacífico & 35.204 & 30,06 & Alto \\
\hline 2015 & Dinamarca & DNK & Europa y Asia central & 48.675 & 54,16 & Alto \\
\hline 2015 & Emiratos Árabes Unidos & ARE & Oriente Medio y Norte de África & 70.255 & 25,51 & Alto \\
\hline 2015 & Eslovenia & SVN & Europa y Asia central & 31.468 & 45,85 & Alto \\
\hline 2015 & España & ESP & Europa y Asia central & 34.818 & 43,25 & Alto \\
\hline 2015 & Estados Unidos & USA & Norteamérica & 56.469 & 35,64 & Alto \\
\hline 2015 & Estonia & EST & Europa y Asia central & 28.689 & 37,36 & Alto \\
\hline 2015 & Finlandia & FIN & Europa y Asia central & 42.071 & 55,63 & Alto \\
\hline 2015 & Francia & FRA & Europa y Asia central & 40.469 & 56,53 & Alto \\
\hline 2015 & Grecia & GRC & Europa y Asia central & 26.697 & 53,14 & Alto \\
\hline 2015 & $\begin{array}{l}\text { Hong Kong, Región } \\
\text { Administrativa Especial }\end{array}$ & $\mathrm{HKG}$ & Asia oriental y el Pacífico & 57.072 & 19,10 & Alto \\
\hline 2015 & Hungría & HUN & Europa y Asia central & 26.148 & 47,53 & Alto \\
\hline 2015 & Irlanda & IRL & Europa y Asia central & 69.394 & 28,56 & Alto \\
\hline 2015 & Islandia & ISL & Europa y Asia central & 47.500 & 41,82 & Alto \\
\hline 2015 & Israel & ISR & Oriente Medio y Norte de África & 35.889 & 39,47 & Alto \\
\hline 2015 & Italia & ITA & Europa y Asia central & 36.640 & 50,32 & Alto \\
\hline 2015 & Japón & JPN & Asia oriental y el Pacífico & 40.717 & 38,56 & Alto \\
\hline 2015 & Letonia & LVA & Europa y Asia central & 24.510 & 37,19 & Alto \\
\hline 2015 & Lituania & LTU & Europa y Asia central & 28.784 & 33,94 & Alto \\
\hline 2015 & Luxemburgo & LUX & Europa y Asia central & 102.517 & 39,89 & Alto \\
\hline 2015 & Malta & MLT & Europa y Asia central & 35.874 & 38,60 & Alto \\
\hline 2015 & Noruega & NOR & Europa y Asia central & 61.722 & 46,56 & Alto \\
\hline 2015 & Nueva Zelandia & NZL & Europa y Asia central & 37.427 & 35,73 & Alto \\
\hline 2015 & Países Bajos & NLD & Europa y Asia central & 49.528 & 42,65 & Alto \\
\hline 2015 & Polonia & $\mathrm{POL}$ & Europa y Asia central & 26.595 & 39,58 & Alto \\
\hline 2015 & Portugal & PRT & Europa y Asia central & 29.532 & 48,05 & Alto \\
\hline 2015 & Reino Unido & GBR & Europa y Asia central & 41.580 & 41,70 & Alto \\
\hline 2015 & $\begin{array}{l}\text { Región Administrativa Espacial, } \\
\text { Maco China }\end{array}$ & MAC & Asia oriental y el Pacífico & 106.960 & 15,25 & Alto \\
\hline 2015 & República Checa & CZE & Europa y Asia central & 33.469 & 40,34 & Alto \\
\hline 2015 & República Eslovaca & SVK & Europa y Asia central & 29.522 & 42,77 & Alto \\
\hline 2015 & San Marino & SMR & Europa y Asia central & 60.029 & 40,89 & Alto \\
\hline 2015 & Seychelles & SYC & & 27.181 & 29,16 & Alto \\
\hline 2015 & Singapur & SGP & Asia oriental y el Pacífico & 86.139 & 16,52 & Alto \\
\hline 2015 & Suecia & SWE & Europa y Asia central & 47.891 & 47,88 & Alto \\
\hline 2015 & Suiza & CHE & Europa y Asia central & 63.648 & 32,58 & Alto \\
\hline
\end{tabular}

Países incluidos en el análisis de comparación internacional, clasificados por nivel de ingreso en US\$ PPA

\section{Agradecimientos}

Un agradecimiento sincero a la Dra. Ángeles Sanchez, profesora del Departamento de Estructura Económica y Economía del Desarrollo de la Universidad Autónoma de Madrid (UAM) por sus valiosos comentarios y revisiones al trabajo del fin de máster del cual resulta esta publicacion, el mismo se hizo acreedor al Segundo Premio a mejor trabajo de fin de máster en Desarrollo Económico y Políticas Públicas en la UAM en el curso académico 2017/2018.

\section{ReFERENCIAS BibLIOgRÁFICAS}

Altunc, F., \& Aydin, C. (2013). The Relationship between Optimal Size of Government and Economic Growth: Empirical Evidence from Turkey, Romania and Bulgaria. Procedia - Social and Behavioral Sciences(66), 66-75.

Asimakopoulos, S., \& Karavias, Y. (2016). The impact of government size on economic growth. Economics Letters(139), 65-68. 
Juan B. Matute Pacheco. Factores explicativos del crecimiento del sector público. El caso de Ecuad...

Avella, M. (2009). Perspectivas de crecimiento del gasto público en Colombia, 1925-2003 ¿Una visión descriptiva à la Wagner, o à la Peacock y Wiseman? Revista de Economia Institucional, 11(20), 83-137.

Berry, W., \& David, L. (Noviembre de 1984). The Measurement of Government Size: Implications for the Study of Government Growth. (T. U. Association, Ed.) The Journal of Politics, 46(4), 1193-1206.

Boix, C. (2011). Democracy, Development, and the Public Sector. (M. P. Association, Ed.) American Journal of Political Science, 45(1), 1-17.

Cameron, D. (1978). The Expansion of the Public Economy: A Comparative Analysis. The American Political Science Rev, 72(4), 1243-1271.

Cavicchiolia, M., \& Pistoresi, B. (2016). Testing threshold cointegration in Wagner's Law: The role of military spending. Economic Modelling, 59, 23 -31.

Foxley, A. (2012). La trampa del ingreso medio. El desafí de esta década para América Latina. Santiago de Chile: DIMACOFI.CEPLAN.

Garand, J. (1988). Explaining Government Growth in the U.S States. The American Political Science Review, 82(3), 837-849.

Gujarati, D. N., \& Porter, D. C. (2010). Econometría (Quinta Edición ed.). México: McGRAW-HILL/ INTERAMERICANA.

Hibbs, D. (1976). Industrial Conflict in Advanced Industrial Societies. (A. P. Associ, Ed.) The American Political Science Review, 70(4), 1033-1058.

Hibbs, D. (1977). Political Parties and Macroeconomic Policy. (A. P. Association, Ed.) The American Political Science Review, 71(4), 1467-1487.

Homi, K., \& Gill, I. (2007). An East Asian reinaissance: Ideas for economic growth. Washington, DC: World Bank.

Jaén, M. (2004). Evolución contemporánea del Gasto Público en España. Almería: Servicio de Publicaciones de la Universidad de Almería.

Jara, C., \& Umpierrez de Reguero, S. (2014). Evolución del Sector Público Ecuatoriano desde 1998 a 2013. Revista Enfoques: Ciencia Politica y Administración Pública(22), 131-147.

Jetter, M., \& Parmeter, C. (2014). Trade Openness and bigger governments: The role of country size revised. European Journal of Political Economics (37), 49-63.

Magazzimo, C. (2012). Wagner vs Keynes: Public spending and nacional income in Italy. Journal of Policy Modeling(34), 890-905.

Narayan, S., Narayan, B., \& Kumar, P. (2012). Evidence of Wagner's Law from Indian states. Economic Modelling, 29, 1548 - 1557.

Ochando, C. (1999). El Estado del Bienestar: Objetivos, modelos y teorias explicativas. Barcelona: Ariel S.A.

Pickering, A., \& James, R. (2011). Ideology and the Growth of Government. The Review of Economics and Statistics, 93(3), 907-919.

Ram, R. (1987). Wagner's Hypothesis in Time-Series and Cross-Section Perspectives: Evidence from "Real" Data for 115 Countries. The Review of Economics and Statistics, 9(2), 194-204.

Rodriguez, D., Vanegas-Matínez, F., \& Lima, S. (2013). La ley de Wagner versus la hipótesis keynesiana: El caso de México, 1950-2009. Investigación Económica (283), 69-98.

Santos, A. (2014). A Time Series and Panel Analysis of Government Spending and National Income. Munich Personal RePEc Archive (MPRA)(56994).

Sideris, D. (2007). Wagner's Law In 19th Century Greece: A Cointegration and Causality Analysis. Working Paper(64).

Weil, D. N. (2006). Crecimiento Económico. Madrid: Pearson Education. 


\section{Notas}

[1] Fuente: Mauro et al. 2013. “A Modern History of Fiscal Prudence and Profligacy," IMF Working Paper No. 13/5, International Monetary Fund, Washington, DC; Banco Mundial.

[2] Fuente: Banco Central del Ecuador. Noventa años de información Estadística. Series históricas 1927-2017.

(C) Universidad de Cuenca 2019

CC BY-NC-SA

INFORMACIÓN ADICIONAL

Código JEL:: F43, D74 